\section{In-practice practical approach to surgical rehabilitation}

\section{Annelies Scorer and Emily Ní Mhuilleoir}

Physiotherapy aims to maximize normal movement and function through appropriate use of a range of different treatment and management techniques, including manual therapy, electrotherapy, exercise, client education and use of assistive aids. Physiotherapy treatment techniques are being more commonly used on postsurgical patients in veterinary practice, for example following a tibial plateau levelling osteotomy or decompressive spinal surgery. It is important to understand the potential outcomes of these treatments as well as why they are being used, in order to optimize the individual patient's recovery.

In the early postoperative stage (approximately first 1-4 weeks) the main aims of treatment are to aid pain management, manage swelling/inflammation, improve/ maintain range of movement, minimize muscle loss and promote healing or reduce risk of complications. These aims can be achieved with a mixture of 'hands-on' treatments, such as cold compression and owner education to ensure the patient is being managed correctly at home.

In the middle stages of healing and rehabilitation (approximately 4-9 weeks) the main aims of treatment are to promote strengthening, improve flexibility and range of movement and challenge proprioception, while still protecting the surgical site and minimising the risk of re-injury. For example, certain techniques such as stretching and passive range-of-movement exercises may be beneficial alongside active strengthening exercises at this stage.

In the later stages of rehabilitation, the aims are to improve strengthening and proprioception and eventually return to normal functional activities. The aims of the final stages of rehabilitation are very much led by the owner's expectations and the individual patient's normal level of activity and function.

While some patients may benefit from referral to a veterinary physiotherapist, there are a lot of techniques and practical changes that can be carried out in general practice that can hugely benefit these patients, including passive range-of-movement exercises, cold compression, early mobilization and use of assistive aids.

\section{KEY LEARNING OBJECTIVES}

- Be able to define physiotherapy and identify some appropriate treatment techniques used at different stages during surgical rehabilitation

- Understand the aims of these treatment techniques and identify contraindications to treatment

- Apply some of these techniques and practical changes to your own practice

\section{MULTIPLE CHOICE QUESTIONS}

1. When is referral and consent from a veterinary surgeon to carry out physiotherapy on an animal legally required?
(A) Never
(B) Always
(C) Post surgery
(D) In complex cases

2. How can postoperative swelling be reduced?
(A) Cold compress
(B) Rest
(C) Stretching
(D) Heat pack

3. What is one aim of the acute stage of postoperative rehabilitation?
(A) Increase walking time to 1 hour
(B) Prevent re-injury
(C) Carry out sit-to-stand exercises
(D) Stop using any assistive aids

\section{Rehabilitation in the referral setting: when and why is this appropriate}

\section{Annelies Scorer and Emily Ní Mhuilleoir}

In human medicine, physiotherapists have a role in almost all inpatient hospital departments, including aciident and emergency, medicine wards (e.g. cardiac and respiratory patients) and intensive care units (ICUs), as well as providing outpatient services in areas such as rheumatology, pulmonary rehabilitation and stroke rehabilitation. In veterinary medicine, physiotherapy is still a developing role, however we can still play an important part in helping manage a wide range of patients in the acute referral setting as well as offering outpatient rehabilitation services.

In the acute setting, physiotherapy can be beneficial for any patient whose mobility is compromised. This could mean a patient who is completely recumbent or has reduced mobility, regardless of diagnosis or type of surgery they have undergone. It could also be beneficial in patients who have pre-existing conditions, for example, osteoarthritis, where a period of restricted mobility in hospital could negatively affect their function. 


\section{Surgical/Theatre}

The negative effects of immobilization are vast and include changes to circulation, skin integrity, respiratory function, muscle strength, range of movement and overall mobility and function, any of which could result in an increased length of hospital stay. The easiest and most effective way to manage these negative changes is prevention. This is where physiotherapy can be of real value in the acute setting.

There are some absolute contraindications to early mobilization, such as unstable fractures or active bleeding, which are important to understand. However, most patients can be safely mobilized with careful assessment of their needs, following appropriate precautions relating to their condition/surgery and correct handling and use of equipment. The level of mobility carried out will vary from patient to patient, for example, it may not be appropriate to get the patient up and walking outside but it would be appropriate to help them into a supported sitting position.

\section{KEY LEARNING OBJECTIVES}

- Understand the role of physiotherapy and rehabilitation in an acute hospital setting

- Understand how physiotherapy/rehabilitation can be appropriate for any patient whose mobility is compromised, regardless of condition/surgery
- Explain how early intervention and early mobilization can help to reduce the negative side effects of immobility or reduced mobility

\section{MULTIPLE CHOICE QUESTIONS}

1. What are secondary complications of recumbency minimized by?
(A) Early mobilization
(B) Pain management
(C) Weight loss
(D) Crate rest

2. Physiotherapy treatment is not appropriate for which patients?
(A) Those who are non-weight-bearing
(B) Those who have had surgery the previous day
(C) Those who are in ICU
(D) Those who have an unstable fracture

3. Physiotherapy can be beneficial for patients with which conditions?
(A) Orthopaedic conditions only
(B) Neurological conditions only
(C) Impaired mobility regardless of diagnosis
(D) Soft tissue injuries only

\section{Discharge planning and management at home}

\section{Annelies Scorer and Emily Ní Mhuilleoir}

Discharge planning is used within the NHS to help manage hospital flow and bed spaces as well reduce the length of hospital stay for the patient. This involves organizing and planning the date and time of discharge during the preoperative assessment, and making sure the patient has everything they need in place for when they get home. This is obviously done to some extent in every veterinary practice and hospital, however there are some considerations and planning that could be undertaken to make sure this is a smooth process and reduce the risk of complications or readmissions

The key element to successful discharge planning is clear communication with the owner. When planning elective surgery, the owner should be advised at the time of the preoperative consultation about postoperative restrictions and equipment they might need, when they can expect to pick up their pet and any further appointments or costs that may be involved. The owner should be prepared for their pet to have reduced function and mobility for a period of weeks to even months and to provide some degree of nursing care in the acute stages. This process is slightly more complicated when the patient has been admitted for emergency surgery.
However, once the patient is stable, the whole team and owner should be working together to try to discharge the patient as soon as possible. It is important to have realistic expectations about what the owner can and cannot manage with at home, for example, owner's own mobility, working hours, home environment, as these can all cause barriers to successful discharge. The physiotherapy and nursing teams have the potential to play an important role in preparing owners for discharge and to manage their pets at home.

\section{KEY LEARNING OBJECTIVES}

- Explain the barriers and challenges to a successful discharge from hospital

- Understand the role of the physiotherapy and nursing teams in helping to ensure a successful discharge

- Advise owners on ways to manage their pets at home during a period of convalescence

\section{MULTIPLE CHOICE QUESTIONS}

1. When planning elective surgery, when should the owner be first informed about crate resting their pet?
(A) At the postoperative recheck appointment
(B) At the preoperative consultation
(C) At the discharge appointment
(D) Over the phone once they get home with their pet

